\title{
MUC4 activates HER2 signalling and enhances the motility of human ovarian cancer cells
}

\author{
MP Ponnusamy', AP Singh', M Jain', S Chakraborty', N Moniaux' and SK Batra ${ }^{*, 1,2}$ \\ 'Department of Biochemistry and Molecular Biology, University of Nebraska Medical Center, Omaha, NE 68198-5870, USA; ${ }^{2}$ Eppley Institute for \\ Research in Cancer and Allied Diseases, University of Nebraska Medical Center, Omaha, NE 68198-5870, USA
}

The mucin MUC4 is a high molecular weight transmembrane glycoprotein. It consists of a mucin-type subunit (MUC4 $\alpha$ ) and a transmembrane growth factor-like subunit (MUC4 $\beta$ ). The mucin MUC4 is overexpressed in many epithelial malignancies including ovarian cancer, suggesting a possible role in the pathogenesis of these cancers. In this study, we investigated the functional role of MUC4 in the human ovarian cancer cell line SKOV3. The mucin MUC4 was ectopically expressed by stable transfection, and its expression was examined by western blot and confocal microscopy analyses. The in vitro studies demonstrated an enhanced motility of MUC4-expressing SKOV3 cells compared with the vector-transfected cells. The mucin MUC4 expression was associated with apparent changes in actin organisation, leading to the formation of microspike, lammelopodia and filopodia-like cellular projections. An enhanced protein expression and activation of HER2, a receptor tyrosine kinase, was also seen, although no significant change was observed in HER-2 transcript levels in the MUC4-transfected SKOV3 cells. Reciprocal co-immunoprecipitation revealed an interaction of MUC4 with HER2. Further, the MUC4-overexpressing SKOV3 cells exhibited an increase in the phosphorylation of focal adhesion kinase (FAK), Akt and ERK, downstream effectors of HER2. Taken together, our findings demonstrate that MUC4 plays a role in ovarian cancer cell motility, in part, by altering actin arrangement and potentiating HER2 downstream signalling in these cells.

British Journal of Cancer (2008) 99, 520 - 526. doi:10.1038/sj.bjc.66045 I7 www.bjcancer.com

(c) 2008 Cancer Research UK

Keywords: MUC4; ovarian cancer; HER2; motility

Ovarian cancer is a highly lethal disease. It often goes undiagnosed until the last stage when it has already spread to distant sites. The current therapeutic strategies for tackling this malignancy are still inefficient and tumour recurrence of up to $70 \%$ is observed in patients with advanced ovarian carcinoma even after treatment (Auersperg et al, 2001, 2002; Jemal et al, 2007). The mortality from ovarian cancer could be significantly lowered by developing new strategies for the early diagnosis and treatment. A better understanding of the molecular mechanisms underlying its progression and the aggressive phenotype is therefore an urgent requirement for developing novel and effective therapeutic modalities for the treatment of this deadly malignancy.

Mucins are large, heavily glycosylated proteins that provide protection to the luminal epithelial surfaces under normal physiological conditions (Moniaux et al, 2001; Hollingsworth and Swanson, 2004). Alteration in the expression or glycosylation pattern of mucins is often associated with the development of cancer by influencing key cellular functions including growth, differentiation, transformation, adhesion and invasiveness of tumour cells and immune surveillance (Andrianifahanana et al, 2001; Hollingsworth and Swanson, 2004). The mucin MUC4 is a transmembrane mucin that frequently displays an altered expression in many cancers (Carraway et al, 2001b; Hollingsworth and Swanson, 2004; Andrianifahanana et al, 2006).

*Correspondence: Dr SK Batra; E-mail: sbatra@unmc.edu Received 8 May 2008; revised 16 June 2008; accepted 23 June 2008
Previous studies from our laboratory have revealed an aberrant expression of the MUC4 in more than $90 \%$ of ovarian tumours, whereas a very low to no expression was detected in the normal ovaries (Chauhan et al, 2006). An overexpression of MUC4 mRNA has also been previously reported in ovarian cancer (Giuntoli et al, 1998; Lopez-Ferrer et al, 2001). The deduced full-length aminoacid sequence of the MUC4 apoprotein shows the presence of a leader peptide, a serine and threonine-rich non-tandem repeat region, a central large tandem repeat domain containing 16-amino-acid repetitive units, regions rich in potential $\mathrm{N}$-glycosylation sites, two cysteine-rich domains, a putative GDPH proteolytic cleavage site, three epidermal growth factor (EGF)-like domains, a hydrophobic transmembrane domain and a short cytoplasmic tail. The MUC4 mucin is considered to be a homologue of the rat sialo-mucin complex (SMC, rat MUC4) because of similarities in their structural organisation (Komatsu et al, 1999; Moniaux et al, 1999). The SMC is also a heterodimeric glycoprotein composed of an $\mathrm{O}$-glycosylated mucin subunit ASGP1 , and an $\mathrm{N}$-glycosylated transmembrane subunit ASGP-2 containing two EGF domains (Komatsu et al, 2000). The mucin MUC4 also possesses two subunits: an extracellular mucin-like subunit, MUC4, and a growth factor-like transmembrane subunit, MUC4 $\beta$, containing three EGF-like domains (Moniaux et al, 1999, 2001). The overexpression of SMC is associated with the suppression of both cell adhesion and immune killing of tumour cells by altering cell-surface properties and promoting tumour growth in vitro or in vivo (Komatsu et al, 1999, 2000). The SMC acts as a ligand for the receptor tyrosine kinase ErbB2/HER2/neu, via one of its two 
EGF-like domains, and induces its phosphorylation (Komatsu et al, 2001; Carraway et al, 2001b, 2002a,b). Similarly, MUC4 has been shown to be involved in the growth and metastasis of pancreatic cancer cells (Singh et al, 2004; Moniaux et al, 2007). In pancreatic cancer cells, MUC4 induces ultra-structural alterations and promotes tumour cell proliferation and survival and interferes in the interaction of tumour cells with extracellular matrix (Chaturvedi et al, 2007; Moniaux et al, 2007).

In this study, we have investigated the functional consequences of ectopic MUC4 expression in a human ovarian cancer cell line SKOV3. Our studies demonstrate a direct association of the MUC4 mucin with increased motility in ovarian cancer cells. We also show that MUC4 interacts with HER2, enhances its expression and potentiates its downstream signalling.

\section{MATERIALS AND METHODS}

\section{Cell culture and stable transfection of MUC4-expression construct}

The MUC4 cDNA is very large $(29 \mathrm{~kb})$; therefore, it was difficult to clone the entire coding sequence into any available expression vector. Thus, we reduced the size of the repetitive sequence (by decreasing the number of tandem repeats) and generated an MUC4-expression plasmid, which encodes a protein of $320 \mathrm{kDa}$ (Moniaux et al, 2007). The SKOV3 ovarian cancer cells were procured from ATCC (Manassas, VA, USA) and cultured in DMEM medium supplemented with $10 \%$ foetal calf serum and antibiotics. The cultures were maintained in a humidified atmosphere at $37^{\circ} \mathrm{C}$ with $5 \% \mathrm{CO}_{2}$. The MUC4-expression plasmid and empty vector (pSecTag/MUC4 and pSegTag) were stably transfected in SKOV3 ovarian cancer cells by Fugene (Invitrogen Corp., Carlsbad, CA, USA), following the manufacturer's protocol. The Zeocin-resistant colonies were isolated by the ring cloning method and maintained in medium supplemented with $250 \mu \mathrm{g} \mathrm{ml}^{-1}$ Zeocin (Invitrogen Corp.). Medium was replaced with complete medium without antibiotic supplement at least 5 days before any analysis.

\section{RNA isolation and quantitative RT - PCR for HER2 mRNA}

Transcript levels of HER2 in MUC4-transfected SKOV3 cells were measured by SYBR ${ }^{\circledR}$ Premix Ex Taq ${ }^{\mathrm{TM}}$ kit. cDNA was synthesised using $2 \mu \mathrm{g}$ of total RNA, oligo(dT) $)_{18}$ primer and Superscript RT (Invitrogen Corp.). Quantitative real-time PCR was performed using $1 \mu \mathrm{l}$ of a $1: 5$ dilution of first-strand cDNA using the SYBR Premix Ex Taq kit (Takara Bio, Madison, WI, USA) and primers specific to HER2 and RPL13A on an ABI 7500 Sequence Detection System (Applied Biosystems, Foster, CA, USA). The receptor tyrosine kinase HER2 was amplified using the forward primer $5^{\prime}$ TCA CCT ACA ACA CAG ACA CGT TTG- $3^{\prime}$ and the reverse primer $5^{\prime}$-ATC CCA CGT CCG TAG AAG GTA-3', whereas RPL13A was amplified using the forward primer $5^{\prime}$-ATC GTG GCT AAA CAG GTA CTG- $3^{\prime}$ and the reverse primer $5^{\prime}$-GCA CGA CCT TGA GGG CAG $C-3^{\prime}$. The efficiency of the PCR and the specificity of primers were determined using serial dilutions of template cDNA and dissociation curve analysis. Each cDNA sample was used in triplicate, and a reaction without any $\mathrm{CDNA}$ was used as negative control. The levels of HER2 mRNA were then normalised to the RPL13A mRNA levels, and results were graphically represented as a fold difference in HER2 mRNA level in MUC4-overexpressing versus empty-vector-transfected cells.

\section{Immunoprecipitation and immunoblot analysis}

Cells were grown to $70-80 \%$ confluence in a $5 \% \mathrm{CO}_{2}$ incubator at $37^{\circ} \mathrm{C}$. Cells were rinsed once with ice-cold phosphate-buffered saline (PBS) containing $1 \mathrm{~mm}$ sodium orthovanadate and lysed in Triton X-100 lysis buffer ( $150 \mathrm{~mm} \mathrm{NaCl,} 50 \mathrm{~mm}$ Tris- $\mathrm{HCl}$ [pH 8.0],
$5 \mathrm{~mm} \mathrm{NaF}, 1.0 \%$ Triton X-100, $1 \mathrm{~mm}$ sodium orthovanadate, $5 \mu \mathrm{g}$ of aprotinin per $\mathrm{ml}$ and $5 \mu \mathrm{g}$ of leupeptin per $\mathrm{ml}$ ) for $25-35 \mathrm{~min}$ at $4{ }^{\circ} \mathrm{C}$. The lysates were centrifuged at $16000 \mathrm{~g}$ for $20 \mathrm{~min}$ at $4{ }^{\circ} \mathrm{C}$. Protein concentrations were measured using the micro-BCA protein estimation kit (Bio-Rad, Hercules, CA, USA). Equal amounts of protein were incubated overnight with anti-MUC4 (mouse monoclonal) or anti-HER2 (Rabbit polyclonal) antibodies in a $500-\mu$ l total volume. Protein $\mathrm{A}+\mathrm{G}$-Sepharose beads (Oncogene Research, Boston, MA, USA) were added to the lysate-antibody mix and incubated on a rotating platform for $3 \mathrm{~h}$ at $4{ }^{\circ} \mathrm{C}$ and washed four times with lysis buffer. The immunoprecipitates or total cell lysates were electrophoretically resolved on a gel. Sodium dodecyl sulphate-agarose (2\%) gel electrophoresis was performed for MUC4 using $20 \mu \mathrm{g}$ protein samples under reducing conditions. For HER2, FAK, Akt, ERK, p38 (rabbit polyclonal) and $\beta$-actin (mouse monoclonal) expression, SDS - PAGE (10\%) was performed under similar conditions. Resolved proteins were transferred on to the PVDF membrane. After quick washing in PBST (PBS and 0.1\% Tween 20), the membranes were blocked in 5\% non-fat dry milk in PBS for at least $2 \mathrm{~h}$ and then incubated with primary antibodies (anti-MUC4, both activated form and total of HER2, FAK, Akt, ERK, p38 and $\beta$-actin). The immunoblots were washed five times $(5 \times 10 \mathrm{~min})$, incubated with anti-mouse (for MUC4 and $\beta$-actin blots) and antirabbit (for HER2, FAK, Akt, ERK and p38 blots) horseradish peroxidase-conjugated secondary antibodies, washed five times $(5 \times 10 \mathrm{~min})$, reacted with enhanced chemiluminescence ECL reagent (Amarsham Bioscience, Buckinghamshire, UK) and exposed to X-ray film to detect the signal.

\section{Confocal immunofluorescence microscopy}

Derived cells and SKOV3 were seeded at low density on sterilised cover slips and allowed to grow for $20 \mathrm{~h}$. Cells were washed with Hanks buffer containing 0.1 M HEPES, and fixed in ice-cold methanol at $-20{ }^{\circ} \mathrm{C}$ for $2 \mathrm{~min}$ and blocked with $10 \%$ goat serum (Jackson Immunoresearch Labs Inc., West Grove, PA, USA) containing $0.05 \%$ Tween-20 for at least $30 \mathrm{~min}$. For Phalloidin staining, cells were fixed in $3.6 \%$ formaldehyde-PBS solution and permeabilised with $0.1 \%$ TritonX-100 in PBS for $20 \mathrm{~min}$ at room temperature. After the blocking step and a quick wash in PBS, cells were incubated with the anti-MUC4 monoclonal antibody, and with anti-HER2 polyclonal antibodies for $60 \mathrm{~min}$ at room temperature. Cells were then washed $(4 \times 5 \mathrm{~min})$ with $\mathrm{PBS}$ containing $0.05 \%$ Tween-20 (PBST) and then incubated with FITC-conjugated anti-mouse and Texas red-conjugated anti-rabbit secondary antibodies (Jackson Immunoresearch Labs Inc., West Grove, PA, USA) for $30 \mathrm{~min}$ at room temperature in the dark. Cells were washed $(5 \times 5 \mathrm{~min})$ again and mounted on glass slides in antifade Vectashield mounting medium (Vector Laboratories, Burlingame, CA, USA). Laser confocal microscopy was performed by using an LSM 510 microscope (Carl Zeiss GmbH, Thornwood, NY, USA). Photographs were taken in each channel separately and digitally merged for co-localisation studies.

\section{Motility assay}

For motility assays, $0.5 \times 10^{6}$ cells were plated in the top chamber of monocoated polyethylene teraphthalate membranes (six-well insert, pore size $8 \mu \mathrm{m}$ ) (Becton Dickinson, San Jose, CA, USA). The cells were incubated for $24 \mathrm{~h}$, and the cells that did not migrate through the pores in the membrane were removed by scraping the membrane with a cotton swab. The migrated cells in the membrane were stained with a diff-Quick cell stain kit, and cells in 10 random fields of view at $\times 10$ magnification were counted and expressed as the average number of cells per field of view. Three independent experiments were performed in each case. The 
data were represented as the average of the three independent experiments with the standard deviation indicated.

\section{RESULTS}

\section{Ectopic expression of the MUC4 mucin in transfectants}

We have screened a large number of human ovarian cancer cell lines for expression of MUC4. Our recent study showed that MUC4 is expressed in above $95 \%$ of ovarian tumours (Chauhan et al, 2006); however, it was not detected in the majority of cell lines that were screened (SKOV3, OVCAR3, OVCAR5, OVCAR8, A2780, COV362.4, COV 664, COV434, KGN, SB247, etc.). To investigate the function of MUC4, the cDNA of MUC4 was stably expressed in SKOV3 cells and transfectants were analysed by immunoblotting (8G7 antibody) and confocal microscopy of the immunostained cells. Expression of MUC4 was observed in the MUC4-transfected pooled population of cells (SKOV3-MUC4) and not in the vector control (SKOV3-Vec) (Figure 1A). CD18/HPAF is a MUC4-expressing pancreatic cancer cell line used as a positive control (Figure 1A). Immunofluorescence confocal microscopy showed the expression and localisation of MUC4 in both the plasma membrane and cytoplasm in more than $90 \%$ of the MUC4transfected cells, whereas the vector-transfected control cells showed no MUC4 staining (Figure 1B). Propedium iodide was used as a nuclear stain.

\section{MUC4 expression enhances the motility of SKOV3 cells and causes a rearrangement of the actin cytoskeleton}

We analysed the cellular motility in both vector-transfected and MUC4-overexpressing cells by using Boyden's chamber assay. The MUC4-transfected cells showed a significant $(P<0.05)$ increase in motility when compared with the vector-transfected cells (Figure 2A). Cell motility is typically associated with the coordinated disassembly and reformation of the cortical actin network (Cunningham et al, 1991, 1992). Polymerisation of globular actin (G-actin) leads to the formation of long fibrous molecules called filamentous actin (F-actin). The localisation and distribution of F-actin was analysed by Phalloidin staining in both MUC4-overexpressing and vector-transfected SKOV3 cells. Confocal microscopy of the Phalloidin-stained cells showed filopodia (white arrow), lammelopodia (green arrow) and microspikes (yellow arrow) in MUC4-transfected SKOV3 cells (Figure 2B). In contrast, the vector-transfected SKOV3 cells showed less staining in the cytoplasm and did not show any cellular outgrowth (Figure 2B). Presence of structures like lammelopodia, filopodia and microspikes may be a cause of increased motility in the MUC4-transfected cells.

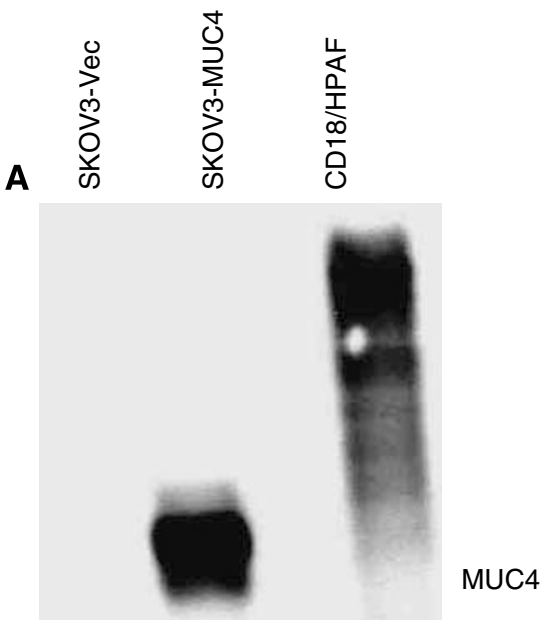

B
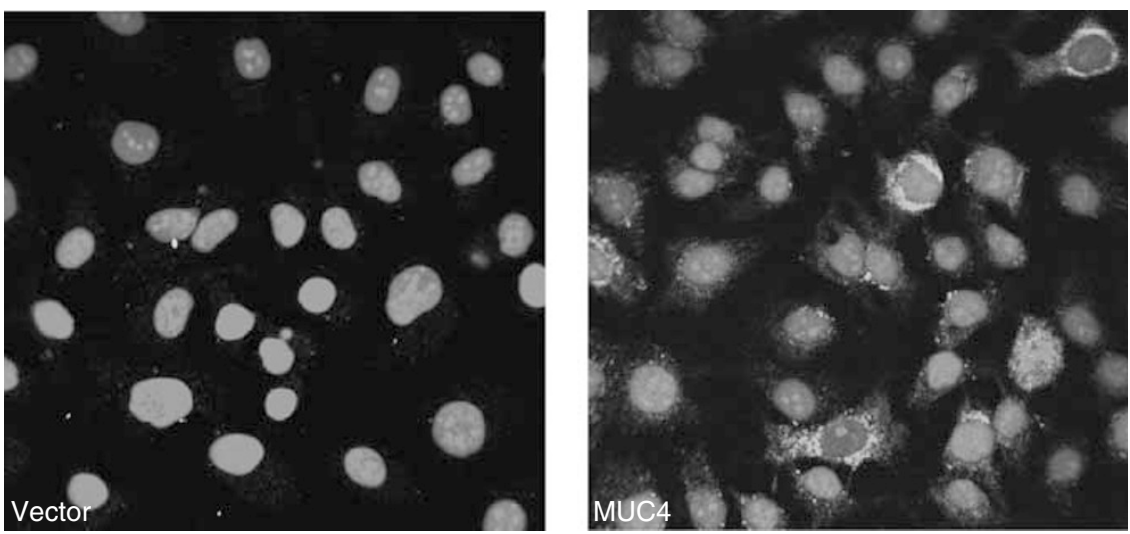

Figure I Expression profile of MUC4 in MUC4-transfected SKOV3 cells. (A) Western blot analysis of MUC4 expression in the vector-transfected (SKOV3-Vec) and MUC4-transfected (SKOV3-MUC4) SKOV3 cell line. The MUC4-expressing pancreatic cancer cell line CDI8/HPAF was used as a positive control. A total of $20 \mu \mathrm{g}$ protein from cell extracts was resolved by electrophoresis on a $2 \%$ SDS-agarose gel, transferred to the PVDF membrane and probed with anti-MUC4 mouse monoclonal antibody (8G7). The membrane was then incubated with horseradish peroxidase-labeled goat anti-mouse immunoglobulin. The signal was detected using an electrochemiluminescence reagent kit. The MUC4 mucin is a high molecular weight glycoprotein and the predicted size of the transfected MUC4 protein is $320 \mathrm{kDa}$. (B) Localisation of MUC4 by confocal microscopy. Cells were grown at low density on sterilised cover slips, washed and fixed in ice-cold methanol at $-20^{\circ} \mathrm{C}$. After blocking with $10 \%$ goat serum, cells were incubated with the anti-MUC4 mouse monoclonal antibody, washed and followed by incubation with FITC-conjugated goat anti-mouse $\lg \mathrm{G}$ ( $\times 630$ original magnification). 

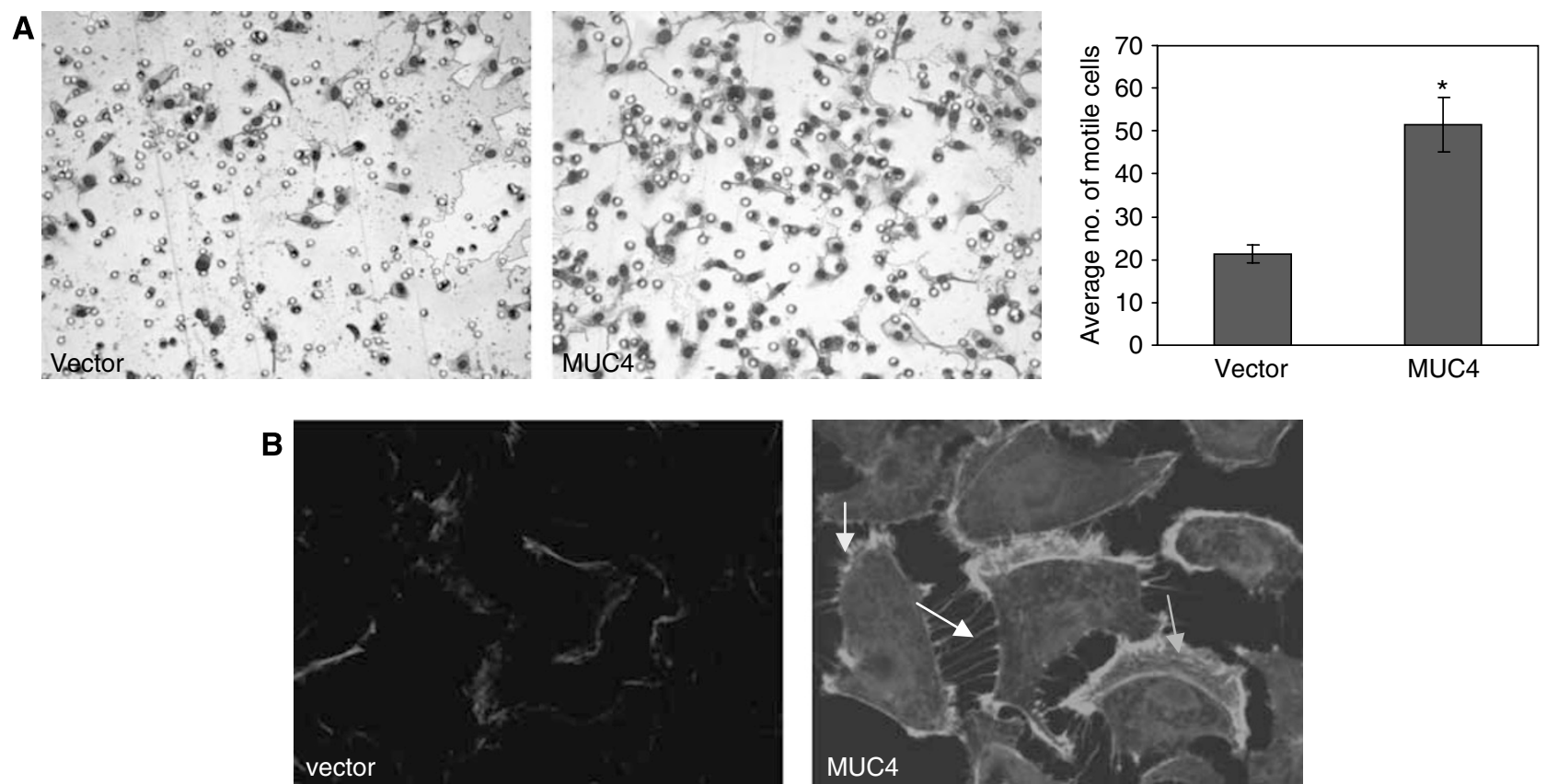

Figure 2 Effect of MUC4 overexpression on the motility and actin organisation of SKOV3 cells. (A) Cells $\left(0.5 \times 10^{6}\right)$ were plated on non-coated membranes for the cellular motility assays. The cells were incubated for $24 \mathrm{~h}$ and allowed to move under a chemotactic effect. The cells that did not migrate through the pores in the membrane were removed by scraping the membrane with a cotton swab, whereas the cells that migrated through the pores were stained. The number of cells traversing the membrane was determined by averaging ten random fields of view at $\times 10$. The data are expressed as the average number of cells per fields of view and is the average of three independent experiments. Cell motility was significantly $(P<0.05)$ increased in the MUC4-transfected SKOV3 cells. (B) Cells were seeded on cover slips and fixed with 3.7\% formaldehyde and permeabilised with 0.1\% Triton X-I00. Cells were stained with $50 \mu \mathrm{g} \mathrm{ml}^{-1}$ fluorescent Phalloidin conjugate solution. SKOV3 cells transfected by MUC4 were associated with the presence of more microspikes (yellow arrows), lammelopodia (green arrows) and filopodia (white arrows)-like cellular projections with dense actin specifically at the cellular protrusions compared with the empty vector control cells (original magnification $1000 \times$ ).

\section{MUC4 interacts with HER2 and increases its expression in SKOV3 cells}

Our previous studies have shown that the knockdown of MUC4 expression significantly reduces the expression level and phosphorylation status of HER2 in the CD18/HPAF pancreatic cell line (Singh et al, 2004). The overexpression of HER2 has also been correlated with increased cellular dynamics and motility in breast cancer cells (Grothey et al, 2000). The expression of HER2 transcript was measured by quantitative real-time reversetranscription assay in MUC4-transfected SKOV3 cells. There was no significant variation in the HER2 transcript level ( $P$-values by applying the $t$-test: 0.889 ) (Figure $3 \mathrm{~A}$ ). Western blot analysis of HER2 in MUC4-transfected SKOV3 cells showed an increased expression of HER2 and its activated form, $\mathrm{pY}^{1248} \mathrm{HER} 2$, as compared with the vector-transfected cells (Figure 3B). Furthermore, our reciprocal co-immunoprecipitation studies indicated that MUC4 and HER2 interact with each other in the MUC4transfected cells (Figure 3C). Confocal microscopy and digital merging of the MUC4- and HER2-immunostained cells demonstrated significant colocalisation of both the proteins in the MUC4transfected SKOV3 cells (Figure 3D).

\section{Activation of HER2 downstream cell signalling in MUC4-transfected SKOV3 cells}

Downstream signalling of HER2 promotes survival and motility of cancer cells (Gabarra-Niecko et al, 2003; Mitra and Schlaepfer, 2006). Focal adhesion kinase is linked to the downregulation of HER2 activity at the cellular periphery, which appears to be important for the formation of motile cells (Mitra and Schlaepfer, 2006). Focal adhesion kinase is a non-receptor tyrosine kinase localised to focal adhesions (Schaller et al, 1992). It is phosphorylated in response to a number of stimuli including the clustering of integrins (Kornberg et al, 1991). Focal adhesion kinase is overexpressed in invasive and metastatic tumours (Owens et al, 1996). An increase level of activated FAK (pY925) was observed in MUC4-transfected SKOV3 cells when compared with the vector-transfected cells (Figure 4). The levels of total FAK, however, remained unchanged in both MUC4- and vectortransfected cells (Figure 4), suggesting that MUC4 activates FAK, either by a direct or an indirect mechanism, which may be responsible to the greater motility observed in the MUC4transfected cells.

The effect of MUC4 on signalling through the AKT and ERK and p38 pathways was also examined in both SKOV3 sublines. The ERK1/2 and Akt signalling pathways play a major role in the migration of cancer cells, respectively. The MUC4-transfected SKOV3 cells showed a significant increase in the levels of activated ERK1/2 and Akt (Figure 4) compared with the vector-transfected cells (Figure 4). The total levels of ERK1/2 and Akt, however, remained unchanged (Figure 4). The increased activation of ERK1/ 2 and Akt may be due to the interaction of the ectopically expressed MUC4 with HER2. The expression level of phospho p38 and total p38 did not show any variation in both the MUC4 and empty vector-transfected cells (data not shown).

\section{DISCUSSION}

The mucin MUC4 is aberrantly expressed in several epithelial malignancies. Our recent studies revealed an overexpression of MUC4 in ovarian (Chauhan et al, 2006) and pancreatic tumours (Andrianifahanana et al, 2001), whereas its expression was downregulated in prostate cancer (Singh et al, 2006). Other studies from our lab have shown that MUC4 is involved in pancreatic 

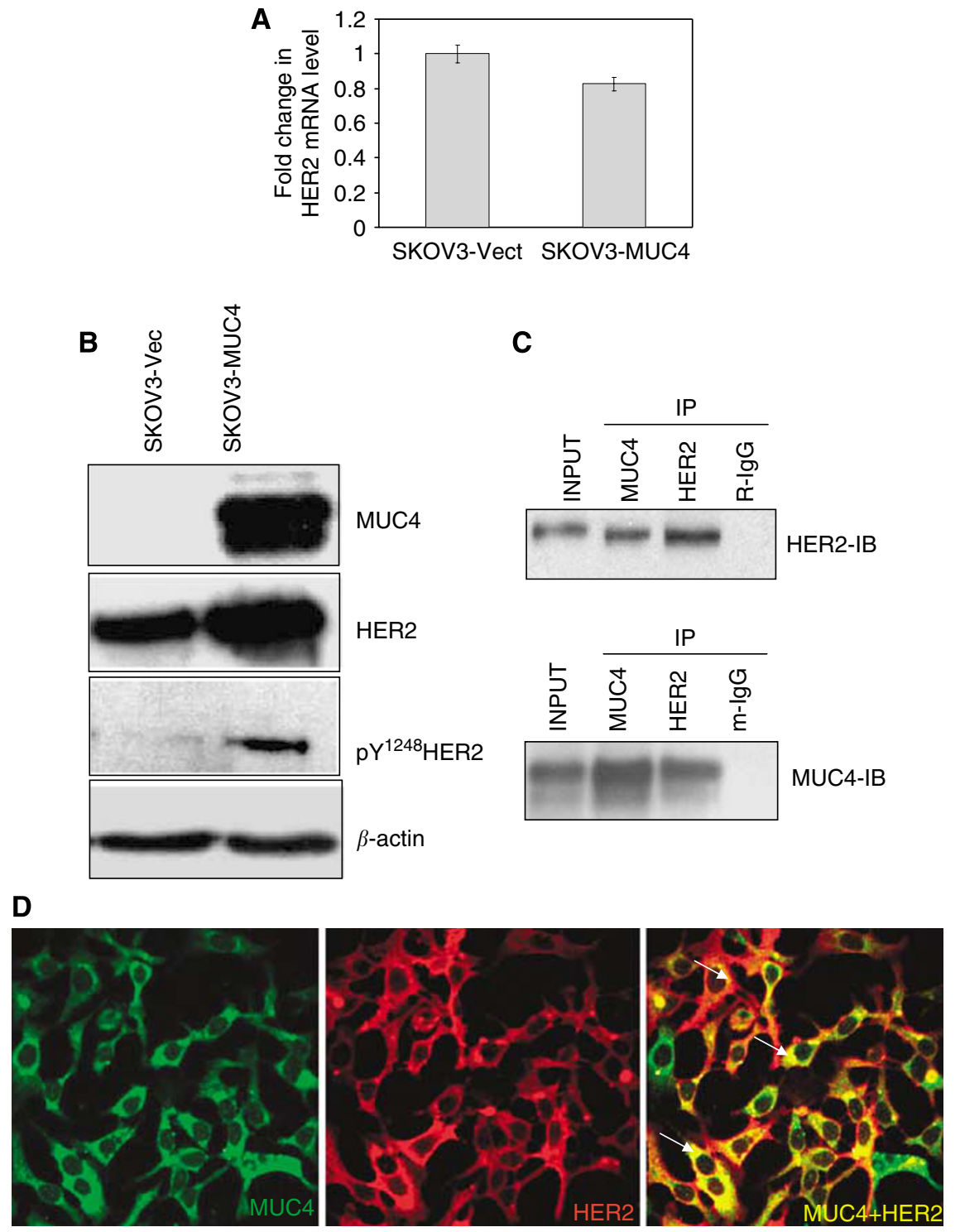

Figure 3 The mucin MUC4 interacts with HER2 and induces the expression of HER2. (A) Estimation of HER2 mRNA levels in MUC4-transfected SKOV3 ovarian cancer cells by real-time RT-PCR. A total of $2 \mu \mathrm{g}$ of RNA was reverse-transcribed and diluted to a total volume of $100 \mu \mathrm{l}(5 \times$ dilution). A total of $\mid \mu \mathrm{l}$ of this cDNA was used in each reaction in a total reaction volume of $10 \mu \mathrm{l}$. Each reaction was plated in triplicate. The results are expressed as the fold changes of HER2 in MUC4-expressed SKOV3 cells relative to that in the vector-transfected cells. No significant change was observed in HER2 transcript levels compared with empty vector-transfected cells ( -values by applying the $t$-test: 0.889). (B) Western blot analysis for HER2 expression and HER2 tyrosine phosphorylation in SKOV3-derived cell lines. A total of $20 \mu \mathrm{g}$ of protein from the cell lysate was resolved by SDS-PAGE, transferred to a PVDF membrane and probed with antibodies against MUC4, HER2, phosphor-Tyr ${ }^{1248}$ HER2 and $\beta$-actin. SKOV3 cells transfected by MUC4 showed an increased expression and tyrosine phosphorylation of HER2 at the Tyr ${ }^{124}$ residue. No phosphorylation of HER2 was seen in the SKOV3-vector control. (C) Reciprocal co-immunoprecipitation analysis to show the interactions between MUC4 and HER2. Lysates from the MUC4-overexpressing SKOV3 cell lines were utilised for immunoprecipitation with MUC4 and HER2 antibodies. The immunoprecipitates were electrophoretically resolved on 2\% SDS-agarose (for MUC4) and 10\% polyacrylamide gel (for HER2), and immunoblotted with anti-MUC4 or anti-HER2 antibodies. The isotype antibodies were used as immunoprecipitation controls. (D) Co-localisation of MUC4 and HER2 in SKOV3 MUC4-transfected cells. Cells were grown at low density on sterilised cover slips, washed and fixed in ice-cold methanol at $-20^{\circ} \mathrm{C}$. After blocking with $10 \%$ goat serum, cells were incubated with the anti-MUC4 mouse monoclonal antibody and anti-HER2 polyclonal antibody followed by incubation with FITC-conjugated goat anti-mouse IgG ( $\times 630$ original magnification). The green staining shows MUC4 expression, red staining shows HER2 expression and the yellow staining shows the co-localisation.

tumour cell growth and metastasis (Singh et al, 2004; Chaturvedi et al, 2007). In this study, we have provided experimental evidence that MUC4 interacts with HER2, potentiates its downstream signalling and enhances the motility of ovarian cancer cells. Our findings provide experimental support for the hypothesis that MUC4 expression is associated with a higher metastatic potential and thereby a poor prognosis in ovarian cancer.

One of the important observations of this study was the interaction of MUC4 and HER2 in ovarian cancer cells, which was also associated with the potentiation of HER2 downstream signalling. Normally, epithelial cells have polarised morphology with apical, basal and lateral regions. The mucin MUC4, wherever present in normal cells, has an apical localisation. However, during the course of malignant transformation, cells lose their polarity, which may allow MUC4 to form novel protein-protein interactions (such as between MUC4 and HER2) with proteins that are normally localised to the basal or lateral surfaces (Ramsauer et al, 2003, 2006). In fact, MUC1, another transmembrane mucin, has 

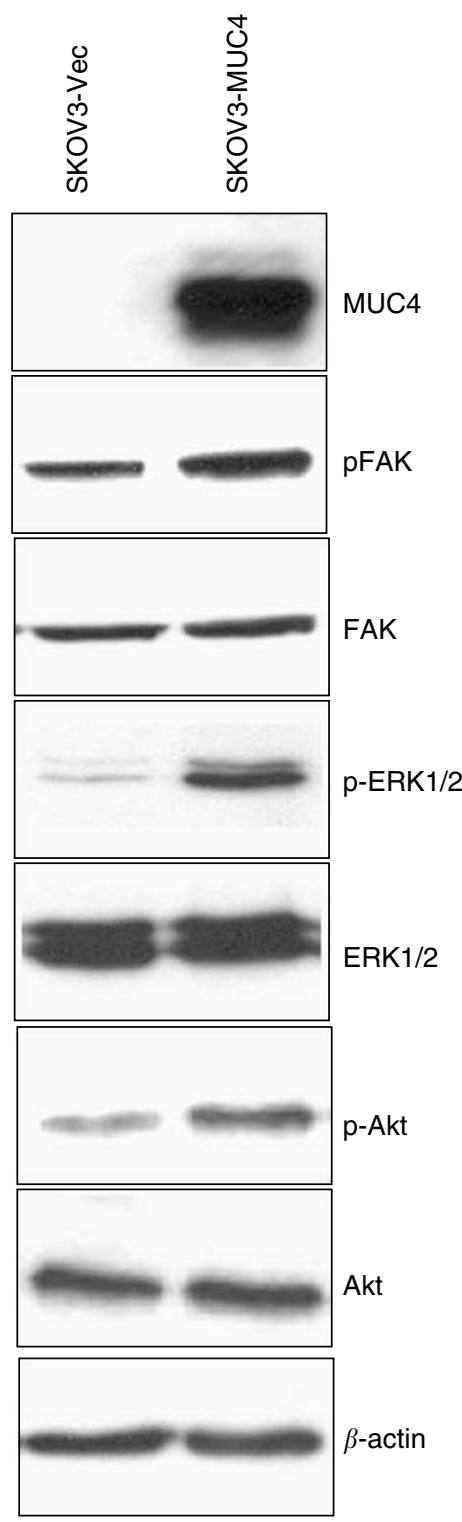

Figure 4 The mucin MUC4 induces HER2 downstream cell signalling in SKOV3 cells. A total of $20 \mu \mathrm{g}$ of cell lysates from SKOV3-vector and SKOV3-MUC4-transfected cells were used for immunoblotting with MUC4, FAK, phosphorylated FAK, ERK, phosphorylated ERK, Akt and phosphorylated Akt antibodies. For MUC4 detection, proteins were resolved on 2\% SDS-agarose gel; other proteins were resolved on $10 \%$ SDS-polyacrylamide gel. $\beta$-actin was used as an internal control. SKOV3 cells transfected by MUC4 clearly showed increased activation of FAK, AKt and ERK in MUC4-transfected SKOV3 cells, whereas the vector-transfected cells showed a lesser activation of FAK, Akt and ERK. The levels of total FAK, Akt and ERK remain unchanged.

been shown to interact with growth factor receptors - such as the ErbB family members, which are otherwise restricted to the lateral and basal cell borders (Schroeder et al, 2001; Scibetta et al, 2001; Canbay, 2003). Moreover, the rat homologue of MUC4 (rMUC4/ SMC) was also shown to interact with ErbB2/neu in multiple cell types (Carraway and Idris, 2001a; Carraway et al, 2001b, 2002a, b). Therefore, our observations on MUC4-HER2 interaction in SKOV3 have a strong functional relevance in ovarian cancer progression. What yet remains to be investigated is the structural basis of this interaction. On the basis of previous findings with rat MUC4, we may speculate a direct interaction of MUC4 with HER2, involving one of the three EGF domains present in the transmembrane (MUC4- $\beta$ ) subunit of MUC4. It is also likely that the cytoplasmic tail of MUC4 or the glycans present on MUC4 may indirectly interact with HER2, as has been shown recently for MUC1-EGFR (Ramasamy et al, 2007). The observation that HER2 expression is increased in MUC4-transfected SKOV3 cells is consistent with our previous finding where we observed a correlative decrease in HER2 expression in antisense-RNAinduced stable MUC4-knockdown cells (Singh et al, 2004). Altogether, our present data and previous observations clearly indicate a role of MUC4 in the regulation of HER2 expression (Singh et al, 2004; Chaturvedi et al, 2007). Notably, our observations are not consistent with previous findings, where rat SMC, although forming a complex with ErbB2 and inducing its phosphorylation, did not affect ErbB2 expression (Carraway et al, 2001b, 2002a,b; Singh et al, 2004). Interestingly, no significant changes in the expression of HER2 transcript were observed in MUC4-transfected SKOV3 cells, suggesting that MUC4-mediated HER2 regulation may occur by post-transcriptional mechanism(s). This is consistent with a recently published report by us in pancreatic cancer cells (Chaturvedi et al, 2008). In a recent study, MUC1 has been shown to inhibit the degradation of ErbB1/HER1 by regulating the receptor trafficking (Pochampalli et al, 2007) and possibly, a similar mechanism may also exist for MUC4-mediated HER2 regulation, which needs to be investigated.

Another important finding of this study was the enhanced cell motility in the MUC4-transfected SKOV3 cells as compared with the vector-transfected controls (Figure 2A). This was also consistent with our previous results, where we observed a three-fold decrease in cell motility upon silencing of MUC4 expression (Singh et al, 2004). Although the exact molecular mechanism for MUC4associated change in cell motility is yet to be deciphered, we predict an important role for augmented HER2 downstream signalling in this process. Exogenous overexpression of HER2 and its activation has been correlated with increased invasiveness and cellular motility (Grothey et al, 2000). Studies on rat MUC4 (SMC) have also demonstrated that MUC4-induced HER2/neu activation may be important in tumour growth and metastasis in rat mammary carcinoma cells (Komatsu et al, 2001; Carraway et al, 2002a). Results from this study have shown an increased activation of FAK in MUC4-transfected SKOV3 cells, whereas the total FAK remained unchanged. Similarly, we also observed an activation of Akt. Previous studies support our observations that HER2 downstream signalling leads to FAK, Akt and ERK activation and is implicated in cell survival and motility (Gabarra-Niecko et al, 2003; Mitra and Schlaepfer, 2006). Recent studies demonstrate that FAK is linked to the downregulation of HER2 activity at the cell periphery, which appears to be important for the formation of motile cells (Mitra and Schlaepfer, 2006). Therefore, the activation of FAK may be related to the increased motility of MUC4-transfected SKOV3 cells. Cell movement is typically associated with a coordinated assembly and disassembly of the cortical actin network (Cunningham et al, 1991, 1992). For migration, a cell requires the formation of cell membrane protrusions containing actin filaments and a continuous process of actin polymerisation near the leading edges (Feldner and Brandt, 2002). It has been shown that the cytoplasmic tail of MUC1 may affect actin polymerisation and increase motility in cancer cells (Parry et al, 1990). In line with this, our data also shows that the actin cytoskeleton is predominantly localised at the leading edges and cellular projections of MUC4-overexpressing SKOV3 cells.

In conclusion, this work is the first report of the direct association of MUC4 with the motile phenotype in ovarian cancer cells. The ectopic expression of MUC4 alters HER2 expression at protein levels, thus potentiate its activation of downstream signalling. Overall, our results demonstrate that MUC4 plays a crucial role in regulating the motility of ovarian cancer cells possibly by altering the expression and activation of HER2 and its downstream signalling. 


\section{ACKNOWLEDGEMENTS}

This work was supported by grants from Department of Defense (OC040592, NIH CA78590) and the Olson Center for Women's Health. We thank Erik Moore (UNMC) for technical support.
We also thank the Molecular Biology Core Facility, UNMC, for oligonucleotide synthesis and DNA sequencing, and Kristi LW Berger (Eppley Institute) for editorial assistance. This work was supported by grants from the US Department of Defense (OC04110) and National Institutes of Health (RO1 CA78590).

\section{REFERENCES}

Andrianifahanana M, Moniaux N, Batra SK (2006) Regulation of mucin expression: mechanistic aspects and implications for cancer and inflammatory diseases. Biochim Biophys Acta 1765: 189-222

Andrianifahanana M, Moniaux N, Schmied BM, Ringel J, Friess $\mathrm{H}$, Hollingsworth MA, Buchler MW, Aubert JP, Batra SK (2001) Mucin (MUC) gene expression in human pancreatic adenocarcinoma and chronic pancreatitis: a potential role of MUC4 as a tumor marker of diagnostic significance. Clin Cancer Res 7: 4033-4040

Auersperg N, Ota T, Mitchell GW (2002) Early events in ovarian epithelial carcinogenesis: progress and problems in experimental approaches. Int $J$ Gynecol Cancer 12: $691-703$

Auersperg N, Wong AS, Choi KC, Kang SK, Leung PC (2001) Ovarian surface epithelium: biology, endocrinology, and pathology. Endocr Rev 22: $255-288$

Canbay E (2003) Erb-B2 homodimerization inhibits MUC1 transcription in cultured human mammary epithelial cells. Cell Biol Int 27: 477-481

Carraway KL, Carvajal ME, Li P, Carraway CA (2002a) ErbB2 and its ligand Muc4 (sialomucin complex) in rat lacrimal gland. Adv Exp Med Biol 506: $289-295$

Carraway KL, Idris N (2001a) Regulation of sialomucin complex/Muc4 in the female rat reproductive tract. Biochem Soc Trans 29: 162-166

Carraway KL, Perez A, Idris N, Jepson S, Arango M, Komatsu M, Haq B, Price-Schiavi SA, Zhang J, Carraway CA (2002b) Muc4/sialomucin complex, the intramembrane ErbB2 ligand, in cancer and epithelia: to protect and to survive. Prog Nucleic Acid Res Mol Biol 71: 149-185

Carraway KL, Price-Schiavi SA, Komatsu M, Jepson S, Perez A, Carraway CA (2001b) Muc4/sialomucin complex in the mammary gland and breast cancer. J Mammary Gland Biol Neoplasia 6: 323-337

Chaturvedi P, Singh AP, Chakraborty S, Chauhan SC, Bafna S, Meza JL, Singh PK, Hollingsworth MA, Mehta PP, Batra SK (2008) MUC4 mucin interacts with and stabilizes the HER2 oncoprotein in human pancreatic cancer cells. Cancer Res 68: 2065-2070

Chaturvedi P, Singh AP, Moniaux N, Senapati S, Chakraborty S, Meza JL, Batra SK (2007) MUC4 mucin potentiates pancreatic tumor cell proliferation, survival, and invasive properties and interferes with its interaction to extracellular matrix proteins. Mol Cancer Res 5: $309-320$

Chauhan SC, Singh AP, Ruiz F, Johansson SL, Jain M, Smith LM, Moniaux N, Batra SK (2006) Aberrant expression of MUC4 in ovarian carcinoma: diagnostic significance alone and in combination with MUC1 and MUC16 (CA125). Mod Pathol 19: 1386-1394

Cunningham CC, Gorlin JB, Kwiatkowski DJ, Hartwig JH, Janmey PA, Byers HR, Stossel TP (1992) Actin-binding protein requirement for cortical stability and efficient locomotion. Science 255: 325-327

Cunningham CC, Stossel TP, Kwiatkowski DJ (1991) Enhanced motility in NIH 3T3 fibroblasts that overexpress gelsolin. Science 251: 1233-1236

Feldner JC, Brandt BH (2002) Cancer cell motility - on the road from c-erbB-2 receptor steered signaling to actin reorganization. Exp Cell Res 272: $93-108$

Gabarra-Niecko V, Schaller MD, Dunty JM (2003) FAK regulates biological processes important for the pathogenesis of cancer. Cancer Metastasis Rev 22: $359-374$

Giuntoli RL, Rodriguez GC, Whitaker RS, Dodge R, Voynow JA (1998) Mucin gene expression in ovarian cancers. Cancer Res 58: 5546-5550

Grothey A, Hashizume R, Ji H, Tubb BE, Patrick Jr CW, Yu D, Mooney EE, McCrea PD (2000) C-erbB-2/HER-2 upregulates fascin, an actin-bundling protein associated with cell motility, in human breast cancer cell lines. Oncogene 19: 4864-4875

Hollingsworth MA, Swanson BJ (2004) Mucins in cancer: protection and control of the cell surface. Nat Rev Cancer 4: 45-60

Jemal A, Siegel R, Ward E, Murray T, Xu J, Thun MJ (2007) Cancer statistics, 2007. CA Cancer J Clin 57: 43-66

Komatsu M, Jepson S, Arango ME, Carothers Carraway CA, Carraway KL (2001) Muc4/sialomucin complex, an intramembrane modulator of
ErbB2/HER2/Neu, potentiates primary tumor growth and suppresses apoptosis in a xenotransplanted tumor. Oncogene 20: $461-470$

Komatsu M, Tatum L, Altman NH, Carothers Carraway CA, Carraway KL (2000) Potentiation of metastasis by cell surface sialomucin complex (rat MUC4), a multifunctional anti-adhesive glycoprotein. Int $J$ Cancer 87: $480-486$

Komatsu M, Yee L, Carraway KL (1999) Overexpression of sialomucin complex, a rat homologue of MUC4, inhibits tumor killing by lymphokine-activated killer cells. Cancer Res 59: 2229-2236

Kornberg LJ, Earp HS, Turner CE, Prockop C, Juliano RL (1991) Signal transduction by integrins: increased protein tyrosine phosphorylation caused by clustering of beta 1 integrins. Proc Natl Acad Sci USA 88: $8392-8396$

Lopez-Ferrer A, Alameda F, Barranco C, Garrido M, de BC (2001) MUC4 expression is increased in dysplastic cervical disorders. Hum Pathol 32: $1197-1202$

Mitra SK, Schlaepfer DD (2006) Integrin-regulated FAK-Src signaling in normal and cancer cells. Curr Opin Cell Biol 18: 516-523

Moniaux N, Chaturvedi P, Varshney GC, Meza JL, Rodriguez-Sierra JF, Aubert JP, Batra SK (2007) Human MUC4 mucin induces ultra-structural changes and tumorigenicity in pancreatic cancer cells. Br J Cancer 97: $345-357$

Moniaux N, Escande F, Porchet N, Aubert JP, Batra SK (2001) Structural organization and classification of the human mucin genes. Front Biosci 6 : D1192-D1206

Moniaux N, Nollet S, Porchet N, Degand P, Laine A, Aubert JP (1999) Complete sequence of the human mucin MUC4: a putative cell membrane-associated mucin. Biochem J 338: 325-333

Owens LV, Xu L, Dent GA, Yang X, Sturge GC, Craven RJ, Cance WG (1996) Focal adhesion kinase as a marker of invasive potential in differentiated human thyroid cancer. Ann Surg Oncol 3: 100-105

Parry G, Beck JC, Moss L, Bartley J, Ojakian GK (1990) Determination of apical membrane polarity in mammary epithelial cell cultures: the role of cell-cell, cell-substratum, and membrane-cytoskeleton interactions. Exp Cell Res 188: $302-311$

Pochampalli MR, el Bejjani RM, Schroeder JA (2007) MUC1 is a novel regulator of ErbB1 receptor trafficking. Oncogene 26: 1693-1701

Ramasamy S, Duraisamy S, Barbashov S, Kawano T, Kharbanda S, Kufe D (2007) The MUC1 and galectin-3 oncoproteins function in a microRNAdependent regulatory loop. Mol Cell 27: $992-1004$

Ramsauer VP, Carraway CA, Salas PJ, Carraway KL (2003) Muc4/sialomucin complex, the intramembrane ErbB2 ligand, translocates ErbB2 to the apical surface in polarized epithelial cells. J Biol Chem 278: $30142-30147$

Ramsauer VP, Pino V, Farooq A, Carothers Carraway CA, Salas PJ, Carraway KL (2006) Muc4-ErbB2 complex formation and signaling in polarized CACO-2 epithelial cells indicate that Muc4 acts as an unorthodox ligand for ErbB2. Mol Biol Cell 17: 2931-2941

Schaller MD, Borgman CA, Cobb BS, Vines RR, Reynolds AB, Parsons JT (1992) pp125FAK a structurally distinctive protein-tyrosine kinase associated with focal adhesions. Proc Natl Acad Sci USA 89: 5192-5196

Schroeder JA, Thompson MC, Gardner MM, Gendler SJ (2001) Transgenic MUC1 interacts with epidermal growth factor receptor and correlates with mitogen-activated protein kinase activation in the mouse mammary gland. J Biol Chem 276: 13057 - 13064

Scibetta AG, Albanese I, Morris J, Cooper L, Downward J, Rowe PP, TaylorPapadimitriou J (2001) Regulation of MUC1 expression in human mammary cell lines by the c-ErbB2 and ras signaling pathways. DNA Cell Biol 20: $265-274$

Singh AP, Chauhan SC, Bafna S, Johansson SL, Smith LM, Moniaux N, Lin MF, Batra SK (2006) Aberrant expression of transmembrane mucins, MUC1 and MUC4, in human prostate carcinomas. Prostate 66: $421-429$

Singh AP, Moniaux N, Chauhan SC, Meza JL, Batra SK (2004) Inhibition of MUC4 expression suppresses pancreatic tumor cell growth and metastasis. Cancer Res 64: 622-630 